Revista Sostenibilidad, Tecnología y Humanismo

16

https://doi.org/10.25213/2216-1872.56

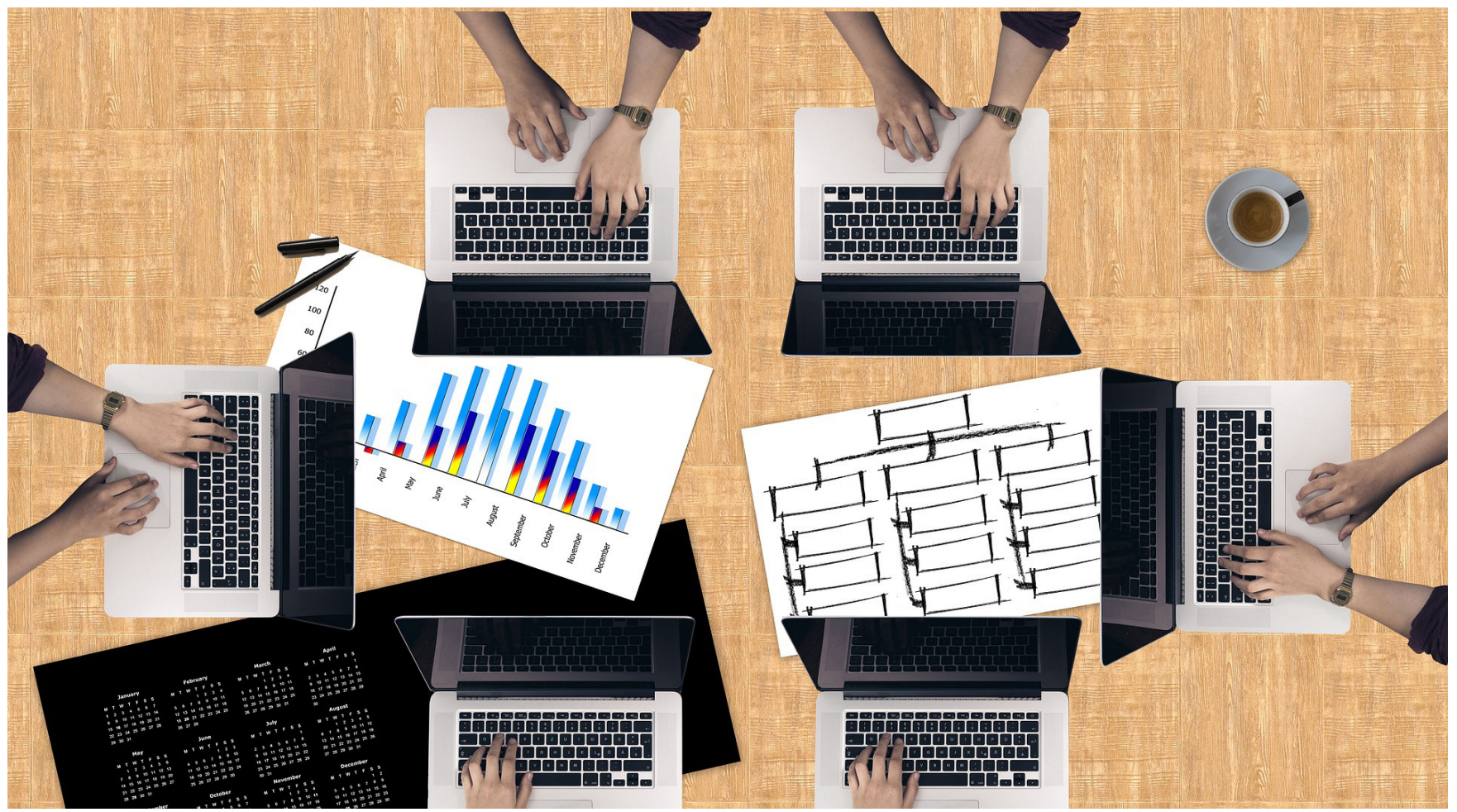

\title{
"El cambio cultural y organizacional, un riesgo prioritario en el montaje de los Proyectos"
}

\section{The cultural and organizational change, a priority risk in the assembly of the Projects"}

Emil Yesid Vélez-López

Especialización en Gerencia, ing.emil.velez@gmail.com, Universidad del Sinú Elías Bechara Zainum, Montería, Colombia. 


\section{Resumen}

En el presente artículo de reflexión se plantean disertaciones del autor, apoyadas en experiencias vividas y en percepciones de otros autores, se habla sobre la importancia del cambio organizacional y de los riesgos que conlleva dicho reto; haciendo énfasis en esto último desde el punto de vista crítico. También se describe cómo cada uno de los posibles errores que se pueden cometer debe ser abordado por los líderes, considerando las múltiples variables que pueden influir en su desarrollo.

Palabras clave: Errores, Cambio Organizacional.

\section{Abstract}

In the present review article author's dissertations are presented, supported by lived experiences and the perceptions of other authors. The importance of organizational change and the risks that this challenge entails are discussed; emphasizing the latter from the critical point of view. It also describes how each of the possible errors that can be committed must be addressed by the leaders, considering the multiple variables that can influence their development.

Keywords: Errors, Organizational Change. 
"El cambio cultural y organizacional, un riesgo prioritario en el montaje de los Proyectos"

\section{8}

\section{Introducción}

Iniciemos haciéndonos la siguiente pregunta ¿Cuáles son los errores que tienen consecuencias peligrosas para el desarrollo de cualquier proyecto que implique un cambio organizacional?. Antes de abordar el tema es fundamental tener en cuenta que: la significación con que entenderemos la palabra "error" es la definida según la real academia de la lengua "Acción desacertada o equivocada", por lo que se convierte, desde nuestro enfoque en: una mala decisión tomada por la organización.

Se debe tener en cuenta que estos proyectos que implican cambio se hacen con buenas intenciones, para mejorar algún aspecto de la organización, el problema es que, cuando se presentan errores se corre un gran riesgo de fracasar. Los errores pueden aparecer en cualquier momento, ya sea en el planteamiento o concepción inicial del proyecto, en su planificación operativa o en el desarrollo de sus actividades. Los errores también podrían presentarse al final del proyecto, al momento de cosechar los resultados se puede "quemar" el pan en la puerta del horno.

Una vez que una organización sufre un percance como los citados anteriormente, cae devastada en su intento, en ocasiones olvida la iniciativa y da vuelta a la página, en otras, con más necesidad que convencimiento, renacen sus esfuerzos como el ave fénix y logra el cometido.

Con respecto a las organizaciones que han logrado éxito en este tipo de aspiraciones, se resalta que el tiempo dedicado, así como el esfuerzo aplicado son considerables y que solo la tenacidad de los líderes logra llevarlos a cabo a buen término.

De acuerdo con Chiavenato [1] "para el logro de un cambio al interior de la organización se debe primariamente modificar el set mental de los miembros de la misma, así, se hace indispensable preparar el ambiente psicológico para que sea adecuado y propicio a la trasformación esperada".

\section{Materiales y métodos}

La investigación parte del apoyo en la revisión documental de diferentes autores con el objetivo de encontrar aspectos teóricos vigentes que puedan orientar las experiencias vividas en cuanto al montaje de Sistemas de Gestión en Organizaciones de diversos tipos. Fue del tipo exploratoria, cualitativa y descriptiva. El carácter exploratorio, manifiesto en el análisis y síntesis en el estudio de los diferentes enfoques y metodologías encontradas con el objetivo de buscar oportunidades de orientar las experiencias. Es cualitativo en la inducción, deducción y análisis histórico lógico para la comprensión de los aportes de diversos autores, en sus perspectivas respecto a las concepciones teóricas desarrolladas. Es descriptivo, en la intencionalidad de analizar con un enfoque sistémico las aplicaciones prácticas de las herramientas propuestas por los autores.

\section{Resultados}

Según el Autor John Kotter [3] Podemos mencionar ocho principales tipos de errores que se presentan con más frecuencia, estos errores pueden llevar al traste con las intenciones de implementar las tan anheladas mejoras procedimentales, en las empresas en las que se ha intervenido se ha visto de cerca muchas situaciones similares.

En la experiencia como implementador y consultor de sistemas de gestión de calidad en diferente tipo de organizaciones es necesario decir que se ha podido observar el ataque de más de uno de estos errores, por lo que se puede dar fe con razón de causa, de su 
actualidad, aunque sea dolorosa su existencia [4].

Con relación a los errores evidenciados encontramoslasiguientedescripción: Podemos comenzar describiendo este primer error: "No establecer un sentido de la urgencia", el cual viene dado por dos grandes razones:

1. La alta dirección ve el proyecto como un requerimiento para cumplir con una obligación legal, la cual consideran molesta y una tarea adicional indeseada, o para cumplir con los requisitos de un cliente, con una licitación, o con otro tipo de oportunidad similar, pero no está convencida de la necesidad real de la organización para llevar a cabo dicho proyecto; no se ve como una necesidad, sino como un deber.

2. La otra, se da cuando se empodera a un ejecutivo de segunda línea, el cual no es lo suficientemente competente para asumir el reto, ni está lo suficientemente convencido de la prioridad de su encargo [5].

En este momento la organización debe contar con un $100 \%$ de convencimiento de la alta dirección, porque a la más pequeña duda de la urgencia de las mejoras buscadas, se puede venir abajo todo lo que se emprenda en estos esfuerzos adicionales de tiempo, dinero y energía.

Error numero 2: "No crear un equipo suficientemente poderoso". Aplica lo dicho anteriormente, con respecto a encargar ejecutivos de segunda línea, esto no debe realizarse delegando todo el poder, ya que la alta dirección debe ser partícipe en los procesos mencionados. Al ser parte activa y que lidera las actividades podrá encargar de las funciones operativas a personas de mando medio, pero sin perder por un momento el control de los cumplimientos de cronogramas, de presupuestos y de resultados [6].
También es importante aclarar que para emprender el cambio tan anhelado es requisito contar con la participación de las personas que son clave en los procesos involucrados en el proyecto, para poder constituir un equipo de trabajo robusto que, de forma mancomunada pueda controlar todas las variables que son prioritarias en la transformación organizacional [7].

Error número 3: "Falta de visión". Se refiere a la claridad que deben tener los objetivos que buscamos con la ejecución del proyecto, a los resultados tangibles que nos pueden dar fe de que se alcanzó el propósito que persigue la organización. Estas intenciones deben ser fáciles de transmitir, lo suficientemente entendibles por todo el personal. En ocasiones se hace difícil trasmitir una visión si esta no es lo suficientemente concreta [8].

Error numero 4: "No saber comunicar la visión". Tal vez la palabra adecuada no sea comunicar, creo que el verbo más acertado es "Comprometer", entendiendo que para comprometeral personal con la visión planeada, la dirección y el equipo implementador deberán asegurarse que se tocan fibras en el alma de los trabajadores, que se pueda palpar el nivel de compenetración de los conceptos que se quieren trasmitir y de la importancia que esto tiene para la organización y para sus colaboradores. Para lograr lo anterior cabe decir que la comunicación es clave, esta debe tener un nivel altísimo de asertividad, usar los canales más apropiados de forma motivadora e innovadora para "atrapar" a su público, el cual es el conjunto de colaboradores de la organización [9].

En cuanto al mensaje que se transmite, debo decir que la coherencia de lo que se postula con relación a lo que se realiza es primordial, para poder convencer y comprometer a las personas la alta dirección y el equipo implementador deberán demostrar que son capaces de ejecutar en sus labores cotidianas 


\section{0}

los cambios que profesan para convertirse en ejes de ese espiral ascendente que quieren introducir en la organización. "Cuando el líder carece de confianza, los seguidores carecen de compromiso." JOHN MAXWELL [10].

Error numero 5: "No remover obstáculos que limitan la nueva visión". Lo primero es identificar estos obstáculos, los cuales pueden ser, por ejemplo:

parte de las labores cotidianas de los colaboradores, es decir la frase famosa "aquí siempre hemos hecho las cosas de la misma manera", dejarse llevar por el status quo, perder el espíritu innovador y negarse a nuevos retos, es uno de los obstáculos más comunes, pero no es el único; encontramos también los obstáculos emanados desde la misma Dirección de la organización, los cuales podrían ser por ejemplo mantener decisiones que en otro momento tuvieron sentido, pero hoy no lo tienen, transformación de hechos coyunturales en estructurales, negándose a evolucionar con la dinámica del mercado [11]. Error numero 6: "No hacer planeación sistemática ni generar victorias a corto plazo". Esto se refiere a la planeación operativa del proyecto, lo cual consta en definir en un cronograma organizado por actividades, responsables y fechas específicas de cumplimiento en un calendario, se debe agregar la columna de presupuesto de inversión a cada rubro definido y los entregables o resultados parciales y finales esperados. Esto se conoce como scheduling, y deberá tenerse en cuenta que todo lo que se defina debe tener una lógica procedimental, donde se pueda retroalimentar periódicamente el avance o estancamiento de las tareas y de esta forma tomar los correctivos que eviten una demora injustificada en las tareas criticas del proyecto, ya que se pondría en riesgo toda su ejecución [12].

Un plazo razonable para rendir cuentas de la ejecución de un proyecto deberá establecerse en forma proporcional a la magnitud, dimensión y duración de las actividades, no podríamos decir que de 12 a 14 meses se entregarán evidencias. Las evidencias de la transformación organizacional deberán responder a la urgencia de la organización, podrían ser entregables e informes mensuales, trimestrales, semestrales, recomendaría que la periodicidad fuera en plazos menores al año, ya que se transformaría en actividades que se pueden perder en el panorama de las tareas cotidianas y recordemos que el proyecto de cambio debe ser muy impactante para que sea un dinamizador de la mejora organizacional [13].

Se pueden incluir reconocimientos, ascensos y dinero para recompensar a las personas. Creo que es interesante para la motivación contar con estas estrategias, pero aún más importante es entender que los ciclos humanos de energía personal y organizacional son cortos y deberán activarse permanentemente en una forma programa da para sacar mayor provecho del largo plazo. Es pensar en el hoy de una forma estratégica para lograr la visión del mañana [14].

¿De qué te sirve administrar bien tu tiempo si tu mente y tu cuerpo no tienen energía para hacer las cosas bien y a la primera? Por más técnicas que tengas de productividad personal, si dejas a un lado la renovación de tu energía, estarás fuera del juego y no llegarás a tu meta (Schwartz, 2003) [15].

Error número siete: "Declarar muy pronto la victoria". En contraste con el punto anterior el cual celebra los triunfos intermedios, declarar la victoria total sin haberla alcanzado es letal para la credibilidad de los grandes proyectos de cambio organizacional, pudiendo dar al traste con todo el esfuerzo invertido $\mathrm{He}$ conocido por ejemplo, empresas que en seis meses logran un certificado de gestión de calidad, y creen que logrando un diploma han llegado al 
culmen de la excelencia, sin entender que este es un logro inicial que simplemente sirve para preparar a la organización para los verdaderos retos de demostrar su mejoramiento continuo sostenido incluyendo los aspectos de rentabilidad [16].

Error número ocho: "No afianzar cambios en la cultura organizacional”: La renovación real que debe sufrir una organización es a largo plazo, cuando las tareas que requieren un esfuerzo explícito del personal por hacer las cosas de manera ejemplar se transforman en rituales automáticos y que son aspectos intrínsecos de la forma de hacer las cosas en los procesos, se transforman en parte de la cultura organizacional [17].

Por último se aconseja que las personas que están proyectadas para asumir cargos en el futuro cercano, sean agentes activos en los macro proyectos y que puedan continuar con el legado impuesto por sus antecesores [18].

\section{Discusión}

Para esta investigación se analizaron diferentes teorías de autores para abordar las posibles fallas en el desarrollo de proyectos y en la gestión del cambio que esta labor conlleva, recogidas en la literatura consultada, que sirvieron de base para la orientación teórica de las experiencias vividas en la Gestión de Proyectos de implementación de sistemas de gestión en empresas del departamento de Córdoba en Colombia.

De su conjugación se obtiene un criterio para el ordenamiento lógico de los posibles errores encontrados al momento de montar proyectos de mejora continua y de cambios organizacionales.

\section{Conclusiones}

Al conocer los ocho principales errores que se pueden presentar en el desarrollo de los proyectos de cambio organizacional, podemos decir que en el momento de emprender la decisión de realizar un proyecto que implique una transformación de la organización, es requerido hacerse una idea de los esfuerzos que debemos hacer para tener bajo control cualquiera de estas manifestaciones, y de esta manera encausar las energías de las personas de la organización, sin dejar de lado los pormenores técnicos del desarrollo de las actividades propias del proyecto en sí. Se plantea el siguiente flujo para controlar lo dicho.

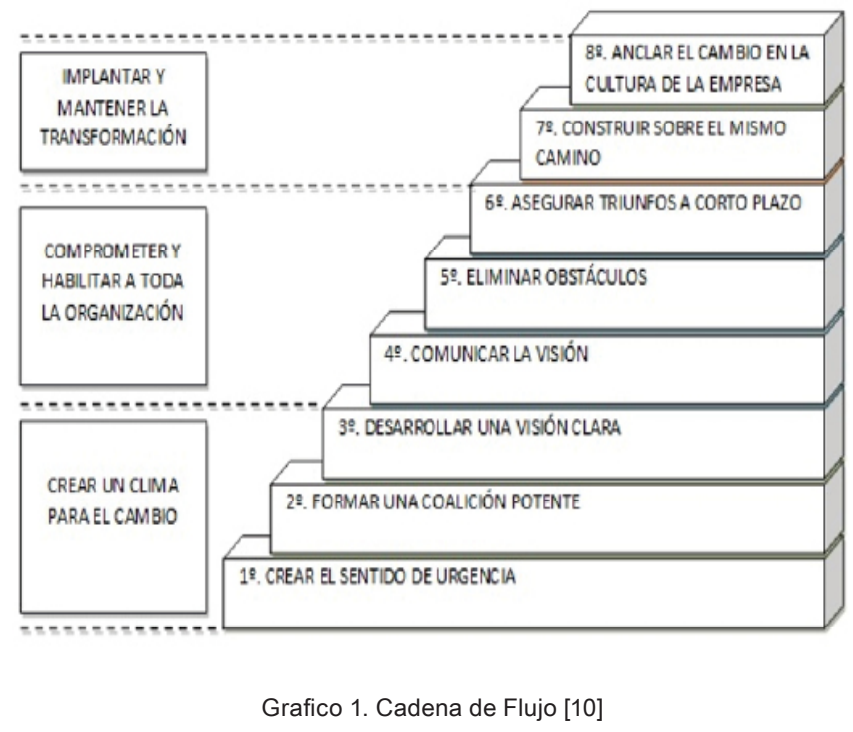

Quedan todavía preguntas por responder, ¿cómo se encuentran nuestras empresas y nuestrosempresarios frenteaestos conceptos?, ya tenemos claro que muchos procesos de cambio no se consiguen por errores en su implementación, pero en general ¿cómo actúan nuestras organizaciones?, ¿será que es un problema de capacitación?, ¿no tendrá algo que ver la actitud de nuestros dirigentes o incluso la cultura? y ¿qué debemos hacer entonces en nuestro papel de directivos? son preguntas que demandan respuestas 
"El cambio cultural y organizacional, un riesgo prioritario en el montaje de los Proyectos"

22

Referencias:

[1]I.Chiavenato,ComportamientoOrganizacional, Mexico DF: MCGRAW-HILL, 2009

[2] K.M. Gutiérrez y G.A. Molinares, Recursos Humanos: Desarrollo organizacional como un proceso de cambio, Managua: Universidad Nacional Autónoma de Nicaragua, 2017

[3] J. Kotter, El líder del cambio, Mexico DF: McGRAW-HILL, 2004

[4] J.L. Schwartz, El poder del pleno compromiso, Madrid: Algaba formación, 2003

[5] A. Suarez Montes, L. Fajardo Cantor, E. Barrientos Monsalve y Y. Rios Epalza, "Estrategias pedagógicas de micro finanzas a los estudiantes del Colegio Municipal Aeropuerto", Mundo Fesc, vol. 4, no 8, pp. 1721,2017

[6] J.L. Sandoval Duque, "Los procesos de cambio organizacional y la generación de valor", Estudios Gerenciales, vol. 30, no. 131, pp. 162171, 2014

[7] J.C. Cachanosky, "Las decisiones empresariales y las predicciones en economía", Revista Libertas 32 Instituto Universitario ESEADE, 2000

[8] J.R. Velásquez-Moreno, G.M. LarrañagaRincón y A. Sarmiento-Linares, "La importancia de formalizar empresa con potenciales emprendedores de la localidad de Bosa, bajo un entorno de globalización económica y desarrollo social", Mundo Fesc, vol. 6 , no 11, pp. 80-90, sep. 2016

[9] J. Hicks, Research, no motion: How the BlackBerry CEOs lost an empire. THE VERGE, 2012
[10] J. Díaz Barrios, "Cambio organizacional: Una aproximación por valores", Revista Venezolana de Gerencia, vol. 10, no. 32, pp. 605-627, 2005

[11] R.E. Quinn, Sabiduría para el cambio, México, D.F.: Prentice-Hall Hispanoamericana, 1997

[12] J. del P. Rodríguez-Tenjo y W. Cruz-Paez, "Planisoft: herramienta web para realizar estimaciones en los proyectos de software", Respuestas, vol. 14, no. 2, pp. 22-31, may 2016

[13] S.P. Robbins, Organizational Behavior: Concepts, Controversies and Applications (6th ed.), New Jersey: Prentice-Hall, 1993

[14] E.H. Schein, Kurt Lewin's change theory in the field and in the classroom: Notes toward a model of management learning, Systems Practice, 1996

[15] P. Senge, A. Kleiner, C. Roberts, R. Ross, G. Roth y B. Smith, La danza del cambio: los retos de sostener el impulso en organizaciones abiertas al aprendizaje. Bogotá: Norma, 2000

[16] D. Tsiantar, Keeping Xerox out of trouble, Time International (Canada edition), 2006

[17] E. Vieira Posada, La globalización en un mundo en transformación, Bogotá: CESA, 2012

[18] A. Wilkinson, Downsizing, rightsizing or dumbsizing? Quality, human resources and the management of sustainability. Total Quality Management, 2005 